\title{
Giardiasis: Conceptos modernos sobre su control y tratamiento
}

\author{
R.C. Andrew Thompson
}

Centro Colaborador de la OMS para Epidemiología Molecular de Infecciones Parasitarias, Facultad de Veterinaria y Ciencias Biomédicas, Universidad de Murdoch, Murdoch, W.A., Australia

\author{
Palabras clave \\ Giardia, impacto clínico • Epidemiología molecular · Transmisión · \\ Patogénesis, Giardia · Tratamiento y control, Giardia
}

\section{Resumen}

Giardia es el patógeno protozoario entérico más corriente en humanos, animales domésticos y animales salvajes. El riesgo máximo de las consecuencias clínicas de la infección por Giardia recae en los niños, particularmente los que habitan en países en vías de desarrollo, en asentamientos comunitarios en condiciones desventajosas. Los estudios de epidemiología molecular han contribuido a aclarar las fuentes de infección y la significación de los reservorios animales para la salud pública. Aunque en la actualidad se conocen aspectos de la patogénesis de la infección por Giardia, estamos todavía muy lejos de poder comprender los factores que predisponen a la enfermedad clínica. Existe la disponibilidad de fármacos eficaces para tratar las infecciones por Giardia, pero sólo pueden actuar como coadyuvantes de los abordajes de salud pública tradicionales en los entornos endémicos donde los niños están frecuentemente infectados.

Copyright $\odot 2008$ Nestec Ltd., Vevey/S. Karger AG, Basel

\section{Introducción}

Las infecciones protozoarias intestinales son corrientes en humanos de todo el mundo [1]. Las infecciones en la infancia, en la gestación y aquellas relacionadas con el SIDA, son las de mayor importancia. La morbilidad y la mortalidad asociadas son elevadas, con más de 58 millones de casos de diarrea protozoaria infantil por año, con respecto a lo cual los costes di- rectos, solamente del tratamiento, se estiman en el orden de 150 millones de USD [1]. El protozoo flagelado, Giardia, es mundialmente el parásito protozoario entérico más frecuente en el humano, y también el parásito entérico más común en los animales domésticos, incluyendo el ganado, los perros y los gatos [1-4].

En los países desarrollados, las infecciones por Giardia son más frecuentes en niños, especialmente en guarderías y viajeros; una incidencia decreciente en estos entornos ha alentado a designar la giardiasis como una enfermedad infecciosa reaparecida en el mundo desarrollado [2-5]. En los países en vías de desarrollo, especialmente Asia, África y Latinoamérica, alrededor de 200 millones de personas presentan giardiasis sintomática y cada año se reportan alrededor de 500.000 casos nuevos [6]. Los niños que viven en comunidades son los más frecuentemente infectados en los países en vías de desarrollo y en grupos en desventaja que habitan en comunidades aisladas, como los aborígenes australianos [1, 2, 7]. Estos niños están a máximo riesgo con respecto a las consecuencias crónicas de la infección por Giardia.

Aunque la OMS ha tomado en consideración los protozoos intestinales durante muchos años, debido a su dinámica patológica muy diferente no han formado parte inicialmente de la Iniciativa de Enfermedades Desatendidas. No obstante, dado que todos tienen un vínculo común con la pobreza, el punto de vista actual consiste en un abordaje integral de todas estas enfermedades. En septiembre de 2004, Giardia fue incluida en la Iniciativa de Enfermedades Desatendidas de la OMS [1].

Los protozoos que forman parte colectivamente del género Giardia han intrigado a biólogos y clínicos durante más de 300 años, precisamente desde que Antony van Leeuwenhoek descubrió por vez primera estos microorganismos [8]. A pesar de

\section{KARGER}

Fax +4161306 1234 E-Mail karger@karger.ch www.karger.com (c) 2008 Nestec Ltd., Vevey/S. Karger AG, Basel 0252-8185/08/0661-0023\$24.50/0

Accesible online en: www.karger.com/ans
R.C. Andrew Thompson

WHO Collaborating Centre for the Molecular Epidemiology of Parasitic Infections School of Veterinary and Biomedical Sciences, Murdoch University Murdoch, W.A. 6150 (Australia)

Tel. +61 893602 466, Fax +61 893606 285, E-Mail a.thompson@murdoch.edu.au 
Tabla 1. Especies/conjuntos de Giardia

\begin{tabular}{ll}
\hline Especie/conjunto & Hospedador \\
\hline G. duodenalis/conjunto A & Humanos y otros primates, perros, gatos, ganadería, roedores y otros mamíferos salvajes \\
G. duodenalis/conjunto B (G. enterica) $)^{1}$ & Humanos y otros primates, perros \\
G. agilis & Anfibios \\
G. muris & Roedores \\
G. psittaci & Aves \\
G. ardeae & Aves \\
G. duodenalis/conjunto C/D $(G . \text { canis })^{1}$ & Perros \\
G. duodenalis/conjunto $\mathrm{F}(G . \text { cati })^{1}$ & Gatos \\
G. duodenalis/conjunto E $(G . \text { bovis })^{1}$ & Ganado vacuno y otra ganadería ungulada \\
G. duodenalis/conjunto $\mathrm{G}(G$. simondi) & Ratas \\
\hline
\end{tabular}

${ }^{1}$ Ver Thompson y Monis [4].

su larga historia, la taxonomía, la patogénesis y la relación con los hospedadores de Giardia no se conocen todavía en todos sus extremos, a pesar de que se trata del más ubicuo de todos los protozoos entéricos que afectan a mamíferos.

\section{Taxonomía}

Los miembros del género Giardia son protozoos flagelados que pertenecen a la clase Zoomastigophorea y al orden Diplomonadida. Suelen afectar a los tubos intestinales de numerosas especies vertebradas [4]. Las afinidades filogenéticas de Giardia han sido objeto de debate durante muchos años. Giardia presenta una organización intracelular muy simple y se ha propuesto que representa un linaje eucariótico de ramificación temprana, que diverge antes de la adquisición de mitocondrias [9]. En consecuencia, Giardia ha llegado a ser un microorganismo clave en los intentos de comprender la evolución de las células eucarióticas [4].

La reciente aplicación de herramientas moleculares, basadas en la RCP, ha permitido la determinación de las relaciones genéticas de una gama de 'cepas' de Giardia morfológicamente idénticas [4, 10-12]. En consecuencia, ahora están identificados un gran número de especies y genotipos de Giardia que difieren principalmente en su gama de hospedadores. La taxonomía actual de Giardia se recopila en la tabla 1 y ha sido revisada extensamente $[4,11,13]$. La nomenclatura más ampliamente aceptada en la actualidad con respecto a los genotipos que han sido caracterizados es 'conjunto o colección', si bien se ha propuesto una taxonomía revisada $[4,11]$. Algunas especies y genotipos/conjuntos parecen estar limitados a especies o tipos de hospedadores particulares (por ejemplo, Giardia conjuntos C/D [G. canis] y E [G. bovis] en perros y ganadería, respectivamente (tabla 1), mientras que otros tienen amplias gamas de hospedadores, incluyendo a humanos (por ejemplo, $G$. duodenalis conjuntos A y B; (tabla 1) y poseen, en consecuen- cia, significación zoonótica. G. duodenalis (sinónimos: G. intestinalis; G. lamblia) es la única especie hallada en el humano.

\section{Ciclo vital}

Giardia posee un ciclo vital bifásico muy simple [14]. El microorganismo produce quistes ambientalmente resistentes, que son vaciados en las heces e iniciarán la infección si son ingeridos por otro hospedador. La exposición, primero a un medio ácido en el estómago y luego a sales biliares en el intestino delgado proximal, estimula la liberación de trofozoitos a partir de los quistes, que cuando se acoplan a la superficie mucosa del intestino delgado y la colonizan se multiplican rápidamente por fisión binaria asexuada. A medida que los trofozoitos pasan a través del intestino delgado se enquistan y pasan a las heces. Los quistes vaciados en las heces constituyen la etapa infectiva y son inmediatamente infectivos cuando se ingieren. Los quistes pueden ser transmitidos directamente de un individuo a otro en circunstancias que son propicias para la transferencia fecal-oral, como en las guarderías o en ámbitos en los que existe poca higiene. Los quistes son capaces de sobrevivir prolongadamente en el ambiente, sobre todo cuando los niveles de humedad son suficientes para evitar la desecación. Como tal, Giardia puede ser transmitida mecánicamente a través de alimentos contaminados, moscas o la ingestión de agua contaminada [12].

\section{Diagnóstico}

La microscopía óptica sigue siendo el medio más práctico para el diagnóstico de Giardia en un entorno clínico, mediante técnicas de concentración, como la centrifugación con sulfato de cinc para concentrar los quistes en las muestras fecales 
$[15,16]$. Dado que la excreción de los quistes es esporádica, deben examinarse varias muestras fecales en el curso de cuatro a cinco días. Se dispone de un cierto número de métodos basados en ELISA que detectan coproantígenos. Estos métodos funcionan bien, pero son relativamente costosos. Debido a su coste, la inmunofluorescencia indirecta y la RCP son herramientas de uso predominante en epidemiología e investigación.

La gran ventaja de la microscopía es que no es específica y, en consecuencia, puede detectar otros parásitos que serían importantes en la determinación de la causa de síntomas inespecíficos como la diarrea.

\section{Epidemiología y transmisión}

Un aspecto importante de la epidemiología de las infecciones por Giardia consiste en conocer la gama de hospedadores de diferentes especies y genotipos/conjuntos, de cómo se mantienen en la naturaleza y su potencial de transmisión cruzada $[3,11]$. Esto reviste una especial importancia en la determinación del potencial zoonótico de infecciones por Giardia en animales domésticos $[3,11]$. Giardia se mantiene en diversos ciclos de transmisión que pueden actuar independientemente, por ejemplo, entre humanos, en ganado, perros o animales salvajes. No obstante, no se conocen las circunstancias en las cuales pueden interactuar tales ciclos, sobre todo cuando el resultado puede ser una transferencia zoonótica. Al respecto, el establecimiento de una taxonomía correcta ha proporcionado la base para un mejor conocimiento de los vínculos entre las infecciones en animales domésticos y humanos $[4,11,17]$ (tabla 1).

La giardiasis es la enfermedad de transmisión hídrica más frecuentemente diagnosticada y, junto a la criptosporidiosis, es el problema de salud pública más importante de los servicios públicos del agua en países en vías de desarrollo [3, 18, 19]. El ganado infectado ha sido implicado hace mucho tiempo como fuente de la transmisión hídrica de giardiasis $[3,10,20]$. No obstante, se dispone de pocos datos de estudios epidemiológicos moleculares en sentido de responsabilizar a los animales domésticos de ser la fuente original de los brotes de transmisión hídrica, por cuyo motivo la fuente más probable reside en la contaminación con los desechos humanos en las aguas cloacales $[3,17]$.

Aunque los estudios sobre la aparición de los diferentes genotipos de Giardia sirven para realzar el riesgo potencial de salud pública derivado de perros y gatos domésticos, no se dispone de datos sobre la frecuencia de la transmisión zoonótica de Giardia $[3,21]$. Tal información puede obtenerse de estudios epidemiológicos moleculares en los que se efectúa el genotipaje aislador de parásitos a partir de hospedadores vulnerables en focos de transmisión endémicos localizados, o como consecuencia de la vigilancia longitudinal y el genotipaje de casos positivos. En la primera de estas posibilidades, la investigación reciente en focos de transmisión endémicos localizados ha aportado datos en apoyo del papel de los perros en los ciclos de transmisión zoonótica de Giardia, que involucra a humanos y perros domésticos de poblaciones en regiones de cultivo de té de Assam, India, y en comunidades de templos en Bangkok, Tailandia [22, 23]. En ambos estudios, algunos perros y sus propietarios, que compartían el mismo hábitat, demostraron albergar aislados de $G$. duodenalis procedentes de la misma colección. En otros estudios se observó que los genotipos zoonóticos de Giardia pueden aparecer frecuentemente en perros mascotas individuales que viven en zonas urbanas [ver revisión 21].

Aunque los animales pueden actuar como reservorios de la infección por Giardia, que en determinadas circunstancias puede diseminarse al humano, desde un punto de vista clínico la transmisión interhumana directa tiene una importancia máxima, especialmente en situaciones en las que la frecuencia de transmisión es elevada. La transmisión interhumana de Giardia puede aparecer indirectamente a través de la ingestión accidental de quistes en el agua o en alimentos contaminados, o directamente en ámbitos en los que el grado de higiene puede estar menoscabado, como guarderías o entornos de poblaciones de condición económica o social muy baja, donde la frecuencia de la transmisión es elevada y/o las condiciones son propicias a una transferencia directa de persona a persona [2, 7]. En tales circunstancias, cabe la posibilidad de que los niños presenten un riesgo de infección constante, a pesar de que puedan instaurarse intervenciones quimioterápicas [1, 24]. Si los niños están expuestos constantemente se reinfectarán rápidamente, dado que los agentes antigiardiasis carecen de actividad residual. El hecho de que los niños en tales entornos endémicos no parezcan desarrollar resistencia a la infección por Giardia puede deberse a la competencia inmunológica subóptima y/o la infección por diferentes cepas/subgenotipos de Giardia [25]. Sería de esperar que las interacciones competitivas pudieran resultar en el predominio de genotipos particulares de Giardia y la exclusión de otros, pero no parece que éste sea el caso.

Los humanos pueden ser infectados por genotipos de Giardia que pertenecen al conjunto A o al conjunto B $[11,13]$. Se dispone de datos abundantes de diferencias fenotípicas entre estos dos conjuntos en características como el metabolismo y la velocidad de crecimiento [4]. Por lo tanto, se ha propuesto la posibilidad de diferencias en la naturaleza de la infección entre estos dos conjuntos en humanos, que pueden reflejarse en la duración de la infección, la sensibilidad a los fármacos y la virulencia [4]. Aunque se dispone cada vez de más datos en apoyo de estas hipótesis, es imprescindible la realización de estudios epidemiológicos moleculares mejor enfocados. Por ejemplo, en comunidades de cultivos de té en Assam, India, las proporciones de las infecciones por los conjuntos B y A en 18 personas infectadas eran del 61 y el 39\%, respectivamente [22]. En otro estudio realizado en el Reino Unido, en el cual se examinaron 35 muestras clínicas humanas, se halló que el 64\% correspondía al conjunto B y el $27 \%$ al subgrupo genético II del 
conjunto A; el resto correspondía a una mezcla del conjunto B y el grupo genético II del conjunto A [26]. Análogamente, en una encuesta institucional realizada en Australia se halló que infecciones por el conjunto B eran más prevalentes (70\%) que las infecciones por el conjunto A (30\%) [27]. También se comprobó que el genotipo del conjunto B era responsable de un brote en una guardería del Reino Unido, donde 21 de 24 casos (88\%) fueron infectados por este genotipo [26]. El estudio longitudinal, que fue realizado en hospitales de día de Perth, Australia occidental, reveló que era 26 veces más probable que los niños infectados por aislados de Giardia pertenecientes al conjunto A, presentasen diarrea en comparación con los niños infectados por aislados del conjunto B [27]. Por lo tanto, los niños infectados por aislados de Giardia del conjunto B no serán excluidos de estos hospitales de día, dado que la exclusión depende de la aparición de diarrea. Esto explicaría el motivo por el cual las infecciones por el conjunto B son más corrientes en estos entornos. Es probable que los niños afectados de tales infecciones no sean tratados, lo cual también plantea preguntas sobre las consecuencias a largo plazo de este tipo de infecciones crónicas en caso de que persistan y no aparezca una 'resolución espontánea'. Se cree que esto es importante en situaciones en las que los niños infectados presentan condiciones muy desfavorables en términos de nutrición y/o exposición a infecciones entéricas concomitantes por otros parásitos como Hymenolepis y Ancylostoma. Esta es la situación en comunidades aborígenes aisladas en el norte de Australia, donde se admite que las infecciones por Giardia contribuyen a trastornos nutricionales y a un crecimiento deficiente. En dichas comunidades, las infecciones por aislados de Giardia del conjunto B son más corrientes que las infecciones por el conjunto A [25, 28, 29].

En tales entornos comunitarios, los niños infectados por Giardia son tratados con regularidad mediante agentes antigiardiásicos, especialmente nitroimidazoles; sin embargo, los fracasos terapéuticos son corrientes y se exacerban por las elevadas tasas de reinfección como consecuencia de la higiene deficiente y la contaminación ambiental [4, 25]. Una hipótesis que debe ser verificada es si los aislados del conjunto B de Giardia son más persistentes que los del conjunto $\mathrm{A}$, poseen una asociación más íntima a la mucosa intestinal que los aislados del conjunto A y son menos sensibles a los agentes antigiardiásicos. Es imprescindible la realización de estudios longitudinales de los efectos a largo plazo de las infecciones por especies/genotipos/subgenotipos relacionados con los conjuntos de Giardia en poblaciones endémicas [1].

Son necesarias encuestas epidemiológicas moleculares a gran escala adicionales de infecciones por Giardia en humanos. Con los limitados datos actualmente disponibles no es posible determinar la distribución geográfica y la prevalencia de genotipos infectivos humanos. Con tales datos podría existir la posibilidad de determinar la importancia de cualesquiera diferencias relacionadas con las cepas en virulencia.

\section{Patogénesis e impacto clínico}

La patogénesis de la infección por Giardia no se conoce en todos sus extremos. El parásito no es invasivo y vive y se reproduce por multiplicación asexuada en la superficie luminal del intestino delgado de su hospedador vertebrado. Aunque las infecciones por Giardia pueden causar diarrea malabsortiva, los factores asociados con ésta no se conocen todavía a ciencia cierta y gran parte de lo que sabemos sobre la patogénesis está limitada a infecciones experimentales. La patogénesis resulta de la interacción entre productos parasitarios, como las proteinazas que rompen la barrera epitelial, y las respuestas inflamatorias e inmunológicas del hospedador [30-33]. Giardia induce una apoptosis de los enterocitos, asociada a desorganización de las proteínas citoesqueléticas y de la unión compacta de una forma dependiente de la cepa [34]. En las infecciones por Giardia se ha descrito atrofia vellosa, acortamiento difuso de las microvellosidades, reducción de la actividad de disacaridasas, menoscabo de la función de barrera epitelial, aumento de la permeabilidad y apoptosis [35]. Datos recientes muestran que la infección por Giardia puede también causar hipersecreción de iones clorhídricos [36]. Se cree que estos cambios son debidos a una combinación de productos parasitarios, posiblemente una toxina, y factores inmunitarios del hospedador, especialmente los que implican las células CD8+ [35].

La infección sintomática en el humano puede no ser evidente en una proporción significativa de individuos infectados [37] y representa sólo una fracción (20 a 80\%) de todas las infecciones por Giardia copropositivas [38-40]. Aunque los síntomas son muy variables, entre ellos destacan manifestaciones continuas, habitualmente a corto plazo, de diarrea, dolor epigástrico, nausea, vómito y pérdida de peso [5, 14]. Los síntomas aparecen normalmente 6 a 15 días después de la infección y duran 2 a 4 días. Como tal, se supone que la infección se resuelve espontáneamente en más del $85 \%$ de los casos (lo que indica la presencia de defensas eficaces del hospedador, aunque aparecen casos crónicos ocasionalmente en ausencia de inmunodeficiencias manifiestas $[38,39]$.

Los factores de riesgo de la giardiasis clínica, particularmente en humanos, todavía están por resolverse, pero involucran claramente los factores de hospedador y ambientales, así como la cepa, el genotipo y el conjunto del parásito [36]. No obstante, debe efectuarse una distinción entre los efectos de una infección única, que puede dar lugar al clásico episodio a corto plazo de diarrea, y los efectos a largo plazo de la infección por Giardia, especialmente en niños que habitan en entornos donde la frecuencia de transmisión es elevada. Aquí, el cuadro difiere en gran medida. En focos endémicos en los cuales la frecuencia de transmisión es elevada y a menudo se intensifica por el grado de higiene deficiente y la contaminación ambiental, los niños están a riesgo en particular de las consecuencias más graves y a largo plazo de la infección por Giardia, asociadas a desnutrición, deficiencia de micronutrientes, incapacidad de mejorar, anemia ferropriva y función 
cognitiva deficiente [1, 40-43]. Obviamente, en estas circunstancias, el impacto de Giardia será exacerbado por una nutrición deficiente/subóptima e infecciones concurrentes por otros parásitos entéricos, como Hymenolepis nana y Blastocystis. Se requiere la urgente realización de estudios longitudinales sobre el impacto de parásitos entéricos sobre el crecimiento infantil y el desarrollo mental en zonas endémicas [1].

\section{Tratamiento y control}

Se dispone de varios fármacos para tratar las infecciones por Giardia en humanos. Entre estos destacan los siguientes: metronidazol, tinidazol y furazolidona (que son nitroimidazoles), albendazol (un bencimidazol) y quinacrina (una acridina sustituta). También se ha demostrado la utilidad de la paromicina en algunas situaciones y se ha propuesto la nitazoxanida como alternativa a los nitroimidazoles convencionales; sin embargo, es imprescindible la realización de más estudios para evaluar por completo su eficacia $[1,14,44,45]$. No obstante, en el momento actual, los nitroimidazoles (metronidazol y tinidazol) y el albendazol son los fármacos de elección para el tratamiento de las infecciones por Giardia [1]. Aunque se han descrito fallos terapéuticos con todos los fármacos corrientemente utilizados, todavía no se ha demostrado convincentemente si se trata de casos de resistencia $[1,14]$. La falta de cumplimiento de los pacientes y los efectos secundarios pueden dar lugar a fallos terapéuticos; además, se dispone de algunos datos sobre la sensibilidad variable entre las cepas de G. duodenalis [1, $14,44,45]$. Las dosis diarias únicas conllevan un mejor cumplimiento (el tinidazol tiene una media vida más prolongada que el metronidazol y es bien tolerado si se ingiere durante las comidas) [1, 14]. El cumplimiento deficiente puede ocasionar resistencia farmacológica y, si bien existen pruebas anecdóticas de resistencia al metronidazol, son imprescindibles estudios adicionales sobre los mecanismos genéticos de resistencia y la sensibilidad al metronidazol vinculada a los conjuntos, así como el desarrollo de resistencia a fármacos múltiples [1]. El albendazol ofrece una alternativa más aceptable a los nitroimidazoles, sobre todo en niños; sin embargo, es necesario administrarlo en varias dosis $[1,45]$.

Se ha propuesto que la terapia probiótica podría ser útil en la prevención de la infección o como coadyuvante del tratamiento de la misma [46]. Se ha comprobado que bacterias comensales pueden determinar la vulnerabilidad y la resistencia a la infección por Giardia en ratones [1, 5, 47]. También se ha demostrado que los lactobacilos probióticos liberan un factor termosensible, de bajo peso molecular, que inhibe la proliferación de los trofozoitos de Giardia en el cultivo in vitro [48]. Estas modernas estrategias terapéuticas justifican una investigación adicional $[1,5]$, en la que podrían probar ser más aplicables y útiles que los fármacos para el tratamiento de niños en regiones endémicas.

Giardiasis: Conceptos modernos sobre su control y tratamiento
El control de las infecciones por Giardia en situaciones endémicas, donde la frecuencia de las reinfecciones es elevada debido a la contaminación ambiental y a la higiene deficiente, representa el reto más candente. Los niños que resultan infectados en estos entornos, especialmente en países en vías de desarrollo y en grupos de condiciones desventajosas, representan el colectivo más importante en términos del impacto clínico de Giardia $[1,2,7]$. En tales circunstancias, conviene debatir si el uso regular de fármacos conlleva algún beneficio. Esto contrasta con la situación de helmintos gastrointestinales, como la uncinaria, con respecto a la cual se ha observado que la quimioterapia regular masiva produce un beneficio considerable en su control $[24,26,45]$. Por ejemplo, en un programa de control duradero, basado en la población, en el que se utilizó un patrón de tratamiento regular durante 5 días con $400 \mathrm{mg}$ de albendazol, en el curso de 6,5 años en una población aislada, se controló eficazmente la uncinaria (Ancylostoma duodenalis); sin embargo, no se detectaron efectos persistentes sobre la prevalencia de Giardia y Hymenolepis [24, 45]. Aunque Giardia fue suprimida perfectamente con la administración de dosis múltiples de albendazol, las dosis únicas, regulares, de albendazol durante 6 meses no suprimieron el parásito a largo plazo. La reinfección por Giardia a través de la vía fecal-oral es rápida en estos entornos, en los que la supervivencia de los quistes es posible, anulando cualesquiera beneficios transitorios de la quimioterapia sin cambios conductuales concomitantes [24, 45]. El tratamiento masivo debe combinarse con programas educacionales apropiados, diseñados para prevenir la reinfección $[1,24]$.

\section{Conclusiones}

En teoría, la prevención y el control de las infecciones protozoarias intestinales es actualmente más factible de lo que jamás había sido antes [1]; sin embargo, el hecho de que se trate o no de una propuesta práctica sigue siendo objeto de debate. La epidemiología molecular ha ejercido recientemente un impacto enorme sobre la taxonomía de Giardia y la caracterización de los agentes etiológicos de la giardiasis en el humano; en este contexto, nos encontramos en una posición mucho más favorable para evaluar los factores de riesgo de la salud pública en términos de nuestro conocimiento de las pautas de transmisión y las fuentes de infección. Existe la necesidad de emprender estudios epidemiológicos moleculares en focos endémicos localizados, bien definidos, particularmente en países en vías de desarrollo y en grupos de condiciones económicas o sociales muy bajas. Nuestro conocimiento de la patogénesis de las infecciones por Giardia ha mejorado y nos encontramos algo más cerca de poder afirmar el por qué la enfermedad clínica aparece en algunos individuos, pero puede no manifestarse en otros. No obstante, es imprescindible la realización de estudios para comprender mejor los desenlaces de la infección dependiente de las cepas, sobre todo en niños. Se dispone de 
fármacos para tratar las infecciones por Giardia; sin embargo, la duda estriba en cuándo utilizarlos y es esencial que su empleo sea complementado por estrategias educativas sanitarias básicas, diseñadas para limitar la frecuencia de la transmisión fecal-oral. Al respecto, tales intervenciones exigirán la cooperación de agencias gubernamentales para mejorar la infraestructura básica en poblaciones que habitan en condiciones económicas o sociales muy bajas.

También se ha propuesto la realización de cursos de formación para profesionales sanitarios sobre protozoos intestinales importantes como Giardia; también debe alentarse la organización de cursos sobre la utilidad de los métodos moleculares para detectar dichos protozoos, destinados a profesionales de atención primaria y guarderías y al personal de laboratorios diagnósticos [1]. Diagnósticos más eficaces llevados a cabo en servicios clínicos y laboratorio incrementarán globalmente la elección del tratamiento y permitirán una reducción de la morbilidad [1]. Además, siempre que se obtengan estadísticas de vigilancia precisas, la opinión sobre la importancia de las enfermedades protozoarias intestinales, sostenida por los gobiernos, las organizaciones no gubernamentales (ONG) nacionales e internacionales y los grupos de promoción, será más realista y propiciará una adjudicación correcta de los fondos para ayudas e investigación [1].

\section{Bibliografía}

1 Savioli L, Smith H, Thompson A: Giardia and Cryptosporidium join the 'Neglected Diseases Initiative'. Trends Parasitol 2006; 22:203-208.

2 Thompson RCA: Giardiasis as a re-emerging infectious disease and its zoonotic potential. Int J Parasitol 2000;30:1259-1267.

-3 Thompson RCA: The zoonotic significance and molecular epidemiology of Giardia and giardiasis. Vet Parasitol 2004;126:15-35.

${ }_{4}$ Thompson RCA, Monis PT: Variation in Giardia: implications for taxonomy and epidemiology. Adv Parasitol 2004;58:69-137.

5 Eckmann L: Mucosal defences against Giardia. Parasite Immunol 2003;25:259-270.

6 World Health Organization: The World Health Report 1996. Fighting Disease Fostering Development. Geneva, WHO, 1996.

7 Hesham MS, Azlin M, Nor Aini UN, et al: Giardiasis as a predictor of childhood malnutrition in Orang Asli children in Malaysia. Trans R Soc Trop Med Hyg 2005;99:686691.

8 Meyer EA: Giardia as an organism; in Thompson RCA, Reynoldson JA, Lymbery AJ (eds): Giardia: from Molecules to Disease. Wallingford, CAB International, 1994, pp 3-15.

\9 Simpson AG, Roger AJ, Silberman JD, et al: Evolutionary history of 'early-diverging' eukaryotes: the excavate taxon Carpediemonas is a close relative of Giardia. Mol Biol Evol 2002;19:1782-1791.

10 Smith HV, Caccio SM, Tait A, et al: Tools for investigating the environmental transmission of Cryptosporidium and Giardia infections in humans. Trends Parasitol 2006;22: 160-167.

-11 Caccio SM, Thompson RCA, McLauchlin J, Smith HV: Unravelling Cryptosporidium and Giardia epidemiology. Trends Parasitol 2005;21:430-437.

12 Traub RJ, Monis PT, Robertson ID: Molecular epidemiology: a multidisciplinary approach to understanding parasitic zoonoses. Int J Parasitol 2005;35:1295-1308.
13 Thompson RCA, Traub RJ, Parameswaran, $\mathrm{N}$ : Molecular epidemiology of foodborne parasitic zoonoses; in Murrell KD, Fried B (eds): Food-Borne Parasitic Zoonoses. Berlin, Springer, in press.

14 Monis PT, Thompson RCA: Cryptosporidium and Giardia-zoonoses: fact or fiction? Infect Genet Evol 2003;3:233-244.

15 Thompson RCA, Reynoldson JA, Mendis AHW: Giardia and giardiasis. Adv Parasitol 1993;32:71-160.

16 Zajac AM, Johnson J, King SE: Evaluation of the importance of centrifugation as a component of zinc sulfate fecal flotation examinations. J Am Anim Hosp Assoc 2002;38: 221-224.

17 Thompson RCA, Palmer CS, O'Handley R: The public health and clinical significance of Giardia and Cryptosporidium in domestic animals. Vet J 2007; E-pub ahead of print.

18 Hunter PR, Thompson RC: The zoonotic transmission of Giardia and Cryptosporidium. Int J Parasitol 2005;35:1181-1190.

19 Levine WC, Stephenson WT, Craun, GF: Waterborne disease outbreaks, 1986-1988. MMWR CDC Surveill Summ 1990;39:1-13.

20 Thurman R, Faulkner B, Veal D, et al: Water quality in rural Australia. J Appl Microbiol 1998;84:627-632.

21 Thompson RCA: Molecular epidemiology of Giardia and Cryptosporidium infections. J Parasitol 2003;89:S134-S140.

22 Leonhard S, Pfister K, Beelitz P, et al: The molecular characterisation of Giardia from dogs in Southern Germany. Vet Parasitol 2007;150:33-38.

23 Traub RJ, Robertson ID, Irwin PJ, et al: Canine gastrointestinal parasitic zoonoses in India. Trends Parasitol 2004;21:42-48.

24 Inpankaew T, Traub R, Thompson RCA, Sukthana Y: Canine parasitic zoonoses in Bangkok temples. Southeast Asian J Trop Med Public Health 2007;38:247-255.
25 Thompson RCA, Reynoldson JA, Garrow SJ, et al: Towards the eradication of hookworm in an isolated Australian community. Lancet 2001;357:770-771.

-26 Hopkins RM, Constantine CC, Groth DA, et al: PCR-based DNA fingerprinting of Giardia duodenalis isolates using the intergenic rDNA spacer. Parasitology 1999;118:531539.

-27 Amar CFL, Dear PH, Pedraza-Díaz S, et al: Sensitive PCR-restriction fragment length polymorphism assay for detection and genotyping of Giardia duodenalis in human feces. J Clin Microbiol 2002;40:446-452.

28 Read C, Walters J, Robertson ID, Thompson RCA: Correlation between genotypes of Giardia duodenalis and diarrhoea. Int J Parasitol 2001;32:229-231.

29 Thompson RCA, Meloni BP: Molecular variation in Giardia and its implications. Acta Trop 1993;53:167-184.

30 Meloni BP, Lymbery AJ, Thompson RCA: Genetic characterization of isolates of Giardia duodenalis by enzyme electrophoresis: implications for reproductive biology, population structure, taxonomy and epidemiology. J Parasitol 1995;81:368-383.

31 Chai JY, Guk SM, Han HK, Yun CK: Role of intra-epithelial lymphocytes in mucosal immune responses of mice experimentally infected with C. parvum. J Parasitol 1999;85: 234-239.

32 Scott KG, Yu LC, Buret AG: Role of CD8+ and CD4+ T lymphocytes in jejunal mucosal injury during murine giardiasis. Infect Immun 2004;72:3536-3542.

33 Scott KG, Logan MR, Klammer GM, et al: Jejunal brush border microvillous alterations in G. muris-infected mice: role of $\mathrm{T}$ lymphocytes and Interleukin-6. Infect Immun 2000;68:3412-3418.

34 Guk SM, Yong TS, Chai JY: Role of murine intestinal intraepithelial lymphocytes and lamina propria lymphocytes against primary and challenge infections with C. parvum. J Parasitol 2003;89:270-275. 
35 Chin AC, Teoh DA, Scott KG, et al: Straindependent induction of enterocyte apoptosis by $G$. lamblia disrupts epithelial barrier function inn a caspase-3-dependent manner. Infect Immun 2002;70:3673-3680.

36 Buret AG: Mechanisms of epithelial dysfunction in giardiasis. Gut 2007;56:328335.

>37 Troeger H, Epple HJ, Schneider T, et al: Effect of chronic Giardia lamblia infection on epithelial transport and barrier function in human duodenum. Gut 2007;56:316-317.

38 Rodriguez-Hernandez J, Canut-Blasco A, Martin-Sanchez AM: Seasonal prevalences of Cryptosporidium and Giardia infections in children attending day care centres in Salamanca (Spain) studied for a period of 15 months. Eur J Epidemiol 1996;12:291-295.
39 Flanagan PA: Giardia - diagnosis, clinical course and epidemiology. A review. Epidemiol Infect 1992;109:1-22.

40 Nash TE, Herrington DA, Losonsky GA, Levine MM: Experimental human infections with Giardia lamblia. J Infect Dis 1987; 156:974-984.

41 Berkman DS, Lescano AG, Gilman RH, et al: Effects of stunting, diarrhoeal disease, and parasitic infection during infancy on cognition in late childhood: a follow-up study. Lancet 2002;359:564-571.

42 Gbakima AA, Konteh R, Kallon M, et al: Intestinal protozoa and intestinal helminthic infections in displacement camps in Sierra Leone. Afr J Med Sci 2007;36:1-9.

43 Hesham MS, Edariah AB, Norhavat M: Intestinal parasitic infections and micronutrient deficiency: a review. Med J Malaysia 2004;59:284-293.
44 Gonen C, Yilmaz N, Yalcin M, et al: Diagnostic yield of routine duodenal biopsies in iron deficiency anaemia: a study from Western Australia. Eur J Gastroenterol Hepatol 2007;19:37-41.

45 Wright JM, Dunn LA, Upcroft P, Upcroft JA: Efficacy of antigiardial drugs. Expert Opinion Drug Saf 2003;2:529-541.

46 Reynoldson JA, Behnke JM, Gracey M, et al: Efficacy of albendazole against Giardia and hookworm in a remote Aboriginal community in the north of Western Australia. Acta Trop 1998;71:27-44.

47 Singer SM, Nash TE: The role of normal flora in Giardia lamblia infections in mice. J Infect Dis 2000;181:1510-1512.

48 Perez PF, Minnaard J, Rouvet M: Inhibition of Giardia intestinalis by extracellular factors from Lactobacilli: an in vitro study. Appl Environ Microbiol 2001;67:5037-5042. 